\title{
Effects of dust layers on thermal emission from airless bodies
}

\author{
Jens Biele ${ }^{1^{*}} \mathbb{D}$, Ekkehard Kührt ${ }^{2}$, Hiroki Senshu ${ }^{3}$, Naoya Sakatani ${ }^{4}$, Kazunori Ogawa ${ }^{5}$, Maximilian Hamm², \\ Matthias Grott ${ }^{2}$, Tatsuaki Okada ${ }^{4}$ and Takehiko Arai ${ }^{6}$
}

\begin{abstract}
We have investigated the influence of thin thermally opaque dust layers on the thermal emission of rocks and regolith and determined the thermal response of these dust-covered surfaces to diurnal insolation cycles. Results are computed for Hayabusa2's target asteroid (162173) Ryugu, which was observed by thermal infrared instruments on the orbiter and in situ. We show that even a very thin $(10.100 \mu \mathrm{m})$ fine-grained porous dust layer with thermal inertia of $25 \mathrm{~J} \mathrm{~m}^{-2} \mathrm{~K}^{-1} \mathrm{~s}^{-1 / 2}$ can have a significant influence on surface temperatures and alter the apparent thermal inertia of the underlying material derived under the simplified assumption of a homogenous half space by more than $20 \%$. The masking of the underlying material is complete at about 1 diurnal skin depth, corresponding to $\sim 10 \mathrm{~mm}$ on Ryugu. Between 0.1 and 1 diurnal skin depths, we find a thermal lag smaller than what would be predicted for a surface consisting of dust only.

If a dust cover were present on Ryugu, this should be clearly visible in the data returned by the orbiter's thermal infrared imager (TIR) and the MASCOT lander's radiometer (MARA), which observed a single boulder at the landing site. However, this appears not to be the case, and dust seems to play a minor role in the thermal emission from the asteroid.
\end{abstract}

Keywords: Thermophysical models, Surfaces, Small bodies, Solar system, Thermal inertia, Dust cover, Layering

\section{Introduction}

The Hayabusa2 spacecraft (Watanabe et al. 2017) arrived at its target asteroid (162173) Ryugu in June 2018 and characterized the asteroid's surface using the spacecraft camera suite (ONC), near infrared spectrometer (NIRS3), and thermal infrared imager (TIR). The MASCOT lander (Ho et al. 2017) explored the surface of Ryugu on Oct 3 , 2018, and the MASCOT radiometer MARA determined local surface brightness temperatures over a full diurnal cycle (Grott et al. 2019). MARA observed a single boulder significantly larger than the MARA field of view of about $10 \mathrm{~cm}$ diameter. Images obtained by the MASCOT camera MasCam (Jaumann et al. 2017) provide context for MARA observations. MasCam images have a pixel resolution of $0.15 \mathrm{~mm}$ at best and close to $0.3 \times 0.7 \mathrm{~mm}^{2}$ inside the MARA field of view. Thermal conductivity of the boulder in the MARA field of view was determined to be close to $0.1 \mathrm{~W} \mathrm{~m}^{-1} \mathrm{~K}^{-1}$, much lower than anticipated for

\footnotetext{
* Correspondence: jens.biele@dlr.de

'DLR - German Aerospace Center, RB-MUSC, 51147 Cologne, Germany

Full list of author information is available at the end of the article
}

competent rock (Grott et al. 2019). Here, we investigate whether the low determined conductivity could be the result of a thin dust layer masking the thermal signature of a much more thermally conductive boulder.

\section{Background}

Vertical layering of thermal or physical properties has been extensively studied for the case of Mars, and it was found that layering can significantly influence the shape of the diurnal (and seasonal) surface temperature curves (Putzig and Mellon 2007).

For Mars, modeling of heterogeneous surfaces (Jakosky 1979) provided qualitative explanations of deviations from a homogeneous surface model, and a dust over duricrust model has been suggested already by Ditteon 1982. To investigate the effects of layering, Mellon and Putzig 2007 employed a modified numerical model of a layered subsurface (see (Mellon et al. 2004)). They used this layered thermal model to compute effective temperatures for idealized layered surfaces and derived apparent thermal inertia to examine its variations. In an effort to 
estimate depth to ground ice for Phoenix Lander site characterization, Titus et al. 2006 fit TES brightness temperature observations to two-layer thermal-model results, yielding estimates of ground-ice depth well within a seasonal skin depth in all three of their study regions; they found a best-fit model with a $58 \mathrm{~mm}$-thick layer of sand-like material (thermal inertia $\Gamma=216 \mathrm{~J} \mathrm{~m}^{-2} \mathrm{~K}^{-1} \mathrm{~s}^{-1 / 2}$ ) over a substrate with high thermal inertia $\left(700 \mathrm{~J} \mathrm{~m}^{-2} \mathrm{~K}^{-1} \mathrm{~s}^{-1 / 2}\right)$.

Ruff and Christensen 2002 studied Martian dust (grain size $\sim 40 \mu \mathrm{m}$ similar to our model dust) but are mostly concerned developing the Martian dust cover index DCI which discriminates dust-covered (typical thicknesses of centimeters to meters) regions from dust-free regions and correlates well with albedo and TI. They note that while albedo is sensitive for the first few micrometers, DCI is sensitive to first tens of micrometers, and TI is sensitive to the first few centimeters on Mars. They speculate (no data or calculations are shown to support this) that an intermediate dust cover of few $10 \mathrm{~s}$ of micrometers to a few millimeters may show up in DCI but not strongly in TI, not fully obscuring, in the TI sense, the underlying materials.

So, for the quantitative application of two-layer models, for Mars, mostly a high TI layer over a low TI subsurface with a maximum $\Gamma$ contrast of $\sim 10: 1$, usually much less, has been studied (Vasavada et al. 2017; Edwards et al. 2018). For the high-over-low $\Gamma$ layering, it is found that generally ground temperatures rise later and more slowly in the morning, peak later, and fall off more slowly (and with a more linear trend) in the afternoon and evening afternoon relative to a homogenous surface with intermediate $\Gamma$ (Vasavada et al. 2017).

The perhaps most interesting work for a general two-layer case on Mars on apparent thermal inertia has been presented by Putzig and Mellon 2007. They allow both high-over-low and low-over-high $\Gamma$ layering and find some regions with evidence for the latter (e.g., dust/duricrust, 1-4 mm thick, $\Gamma$ 56/889) as do Audouard et al. 2014.

For the Moon and Mercury, thermal conductivity and density profiles have been derived from Apollo-era data (e.g., (Keihm and Langseth Jr 1973, Mitchell and De Pater 1994)). In particular, only the e-folding length for the profiles have been determined from observed brightness temperatures (Vasavada et al. 1999, Vasavada et al. 2012, Hayne et al. 2017). For asteroids, telescopic observations indicate that vertical layering may also be present. In particular, thermal conductivity, but also density, seem to increase as a function of depth (Harris and Drube 2016). However, methods employed and results obtained for the Moon and Mercury cannot be directly applied here. Instead, we expand on the work of, e.g., Paige et al. 1994.

\section{Methods/Experimental}

In the following, we will report results for regolith thermophysical properties using the surface thermal inertia, but we want to point out that there are a number of complications which need to be kept in mind when using this quantity. Thermal inertia is defined as

$$
\Gamma=\sqrt{k \rho c_{P}}
$$

where $k$ is thermal conductivity, $\rho$ bulk density, and $c_{P}$ specific heat capacity at constant (zero) pressure. However, using thermal inertia as defined by Eq. (1) only makes physical sense if $k, \rho$, and $c_{P}$ are constant, while it is well known that both $k$ and $c_{P}$ depend on temperature (Sakatani et al. 2017; Biele and Grott 2018). Therefore, $\Gamma$ needs to be interpreted with caution, and it is commonly evaluated at a representative surface temperature to mediate the above limitation.

It is also important to note that while (at given albedo, emissivity, insolation) the surface temperature only depends on $\Gamma$ provided that $k, \rho$, and $c_{P}$ are constant, the temperature-depth profile is determined by the product $\rho c_{P}$ as well as $k$. Therefore, the same $\Gamma$ can result in different temperature profiles, and layered surfaces should be described by $k, \rho$, and $c_{P}$ rather than $\Gamma$. Therefore, while using $\Gamma$ to discuss results, all calculations carried out here use $k, \rho$, and $c_{P}$ as variables.

In order to approximate the surface as a half space, considered particle sizes have to be smaller than the diurnal skin depth (Sakatani and Ogawa 2017). The thermal skin depth is the e-folding depth of the subsurface diurnal or seasonal thermal wave. The diurnal temperature curve probes the subsurface to a depth of the order of a few diurnal thermal skin depth, i.e., a few centimeters for Ryugu (Table 2).

We will study the case of a simple yet instructive 2-layer model, in which we assume a homogenous fine-grained material of very low thermal inertia $\Gamma$ to cover a homogeneous subsurface of much higher $\Gamma$. In the modeling, the temperature dependence of thermophysical properties as well as lateral heterogeneity and roughness effects will be ignored.

The general setup of our model is shown in Fig. 1, and two cases will in general be discussed in the following: first, we will consider the effect of a thin dust layer on the thermal emission of solid bedrock with a thermal conductivity appropriate for carbonaceous chondrites (left hand side of Fig. 1), and second, we will consider the influence of dust covering either porous rock or broken up regolith (right hand side of Fig. 1). While the thermophysical properties (Table 1) of the individual layers will be kept constant in our analysis, the thickness of the dust layer $\delta$ will be varied. 


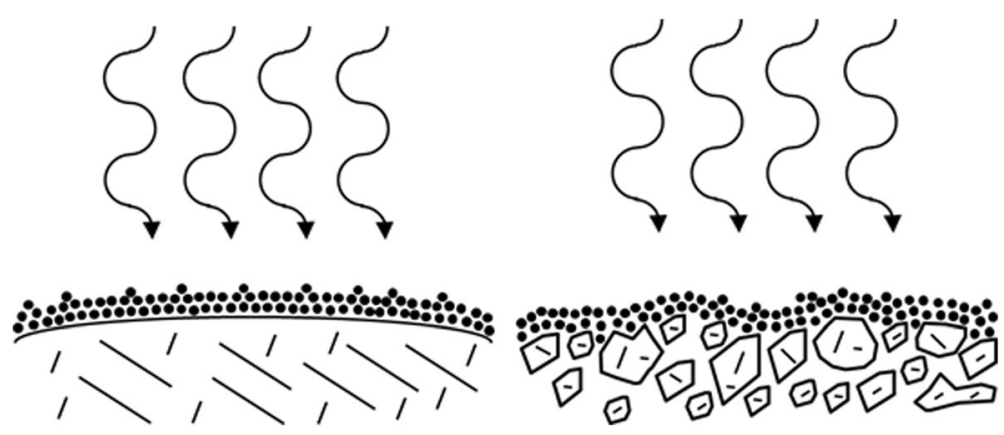

Fig. 1 Left: Two-layer model of an irradiated surface consisting of competent bedrock covered by a thin dust layer. For the rock, a thermal inertia of $1183 \mathrm{~J} \mathrm{~m}^{-2} \mathrm{~K}^{-1} \mathrm{~s}^{-1 / 2}$ will be assumed, while the dust layer has a thermal inertia of $25 \mathrm{~J} \mathrm{~m}^{-2} \mathrm{~K}^{-1} \mathrm{~s}^{-1 / 2}$ (compare Table 1). Average dust thickness is $\delta$. Right: same as left, but for a dust-covered porous rock or coarse regolith with thermal inertia $300 \mathrm{~J} \mathrm{~m}^{-2} \mathrm{~K}^{-1} \mathrm{~s}^{-1 / 2}$

We then solve the one-dimensional heat conduction equation (Delbo et al. 2015), which is given by:

$$
\rho(x) c_{p}(x) \frac{\partial T(t, x)}{\partial t}=\frac{\partial}{\partial x}\left(k(x) \frac{\partial T}{\partial x}\right)
$$

prescribing surface insolation:

$$
\frac{S(1-A)}{r_{h}^{2}} \cos (\theta)=\varepsilon \sigma_{B} T^{4}-\left.k(0) \frac{\partial T}{\partial x}\right|_{x=0}
$$

as the upper boundary condition at $x=0$. Here, $T$ is kinetic temperature, $\sigma_{B}$ the Stefan-Boltzmann constant, $t$ is time, $x$ is depth, $S$ is the solar constant, $A$ is the bond albedo, $r_{h}$ is the heliocentric distance in units of $\mathrm{AU}$, and

\begin{tabular}{|c|c|c|c|c|}
\hline Property & Symbol & Value & Units & Comment \\
\hline Solar constant & $S$ & 1366.1 & $\mathrm{~W} / \mathrm{m}^{2}$ & Solar irradiance at $1 \mathrm{AU}$ \\
\hline Stefan-Boltzmann constant & $\sigma$ & $5.67 \times 10^{-8}$ & $\mathrm{~W} / \mathrm{m}^{2} / \mathrm{K}^{4}$ & \\
\hline \multirow[t]{2}{*}{ Pole orientation } & RA & 96.40101 & {$\left[{ }^{\circ}\right]$} & Right ascension J2000 \\
\hline & DEC & -66.39948 & {$\left[{ }^{\circ}\right]$} & Declination J2000 \\
\hline Rotation period & $P$ & 7.632622 & [h] & \\
\hline Obliquity & I & 171.6428 & {$\left[{ }^{\circ}\right]$} & \\
\hline Orbital period & $T$ & 473.9 & {$[\mathrm{~d}]$} & \\
\hline Absolute time & & & & Oct 03, 2018 \\
\hline Season parameter ${ }^{(1)}$ & $L_{p}$ & 153.5913 & {$\left[{ }^{0}\right]$} & Retrograde spin! \\
\hline Orbit parameters & $e$ & 0.19034 & ( & Subsolar latitude $-5.277^{\circ}$ \\
\hline Perihelion distance & 9 & 0.96315 & {$[\mathrm{AU}]$} & \\
\hline Heliocentric distance & $r_{h}$ & 1.2447 & {$[\mathrm{AU}]$} & Distance to Sun on Oct 3, 2018 \\
\hline Geographical latitude & $\varphi$ & -34.60 & {$\left[{ }^{\circ}\right]$} & MASCOT observation site \\
\hline Specific heat capacity & $c_{p}$ & 700 & {$\left[\mathrm{~kg}^{-1} \mathrm{~K}^{-1}\right]$} & K ((Biele and Grott 2018), average temperature 215 K) \\
\hline Emissivity & $\varepsilon$ & 0.90 & ? & Hemispherical NIR emissivity \\
\hline Bond albedo & A & 0.0178 & ? & \\
\hline Grain density & $\rho_{g}$ & 2100 & {$\left[\mathrm{~kg} \mathrm{~m}^{-3}\right]$} & \\
\hline Bedrock density & $\rho_{b}$ & 2000 & {$\left[\mathrm{~kg} \mathrm{~m}^{-3}\right]$} & Low porosity < 5\% \\
\hline Regolith/porous rocks bulk density & $\rho_{\text {reg }}$ & 1480 & {$\left[\mathrm{~kg} \mathrm{~m}^{-3}\right]$} & Corresponding porosity is $\sim 30 \%$ \\
\hline Dust bulk density & $\rho_{\text {dust }}$ & 366 & {$\left[\mathrm{~kg} \mathrm{~m}^{-3}\right]$} & Corresponding porosity is $83 \%$ \\
\hline Bedrock heat conductivity & $k_{b}$ & 1.0 & {$\left[W^{-1} \mathrm{~m}^{-1}\right]$} & Resulting $\Gamma=1183 \mathrm{~J} \mathrm{~m}^{-2} \mathrm{~K}^{-1} \mathrm{~s}^{-1 / 2}$ \\
\hline Regolith heat conductivity & $k_{\text {reg }}$ & 0.0866 & {$\left[\mathrm{WK}^{-1} \mathrm{~m}^{-1}\right]$} & Resulting $\Gamma=299.5 \mathrm{~J} \mathrm{~m}^{-2} \mathrm{~K}^{-1} \mathrm{~s}^{-1 / 2}$ \\
\hline Dust heat conductivity & $k_{\text {dust }}$ & 0.0025 & {$\left[\mathrm{WK}^{-1} \mathrm{~m}^{-1}\right]$} & Resulting $\Gamma=25.31 \mathrm{~J} \mathrm{~m}^{-2} \mathrm{~K}^{-1} \mathrm{~s}^{-1 / 2}$ \\
\hline
\end{tabular}

Table 1 Parameters used in this study (Wada et al. 2018; Watanabe 2019)

(1)Angle between asteroid's vernal equinox-the ascending node of its equator and orbit-and perihelion, measured in the orbital plane. Orbital elements are 2018 average osculating elliptical elements 
$\theta$ is the local solar zenith angle, where $\cos \theta=0$ during the night. As for the lower boundary condition, we prescribe zero heat flux at a depth of three annual skin depths, and $T(t, x)=0$ is chosen as the initial condition at $t=0$.

The employed algorithm for solving Eqs. (2) and (3) is described in (Kührt and Giese 1989) and provides $T(x, t)$ for independent $k, \rho$, and $c_{p}$ without explicitly using thermal inertia. In our model, depth-dependence is modeled as a step function increase of $k$ und $\rho$ at a given depth $x=\delta$, and we verified that this does not result in any numerical problems. A finite element method from the Fortran NAG library is used for solving the differential equation. Spatial discretization is chosen such that convergence of the results is reached, and at least 10 grid points are located in the dust layer, whereas at least 40 grid points are distributed below. The grid size increases quadratically with depth, where the maximum spacing is limited to be smaller than 3 times the orbital thermal skin depths (i.e., up to $9 \mathrm{~m}$ ). We found that the precise form of the depth grid is not important, as long as spatial and temporal convergence is achieved.

It is well known (e.g., Pelivan 2018) that the number of rotations (or orbits) needed for convergence is a function of $\Gamma$, the daily insolation and the choice of initial conditions. To achieve convergence, calculations were performed for at least half an orbital period, starting from aphelion. Heliocentric distance is changed stepwise, at midnight. The convergence in depth and space was then tested, and it was found that using half the normally used depth step size does not result in a significant change of the obtained temperature profiles. We therefore estimate that the systematic numerical uncertainty of the surface temperatures is smaller than $0.1 \mathrm{~K}$.

The global and orbital parameters used in this study are summarized in Table 1, taken from Wada et al. 2018 and recent Hayabusa2 mission results ((Sugita et al. 2019, Watanabe et al. 2019)).

For the material parameters, we use realistic values either observed for Ryugu or based on measurements of carbonaceous chondrites and the lunar regolith. For the grain or bedrock density, we use an average of the values reported for porous $\mathrm{CI}$ and $\mathrm{CM}$ meteorites (Consolmagno et al. 2008), while the porosity of porous rock or coarse regolith is consistent with either the estimated rock porosity $(\sim 30 \%)$ from MARA measurements (Grott et al. 2019) or a random close packing of polydisperse, slightly irregular, $1 \mathrm{~cm}$ diameter particles, giving the same thermal conductivity than the porous rock.

For the dust fraction, a thermal inertia $\Gamma \approx 25$ to $31 \mathrm{~J}$ $\mathrm{m}^{-2} \mathrm{~K}^{-1} \mathrm{~s}^{-1 / 2}$ has been assumed, representative, e.g., of the uppermost lunar surface with grain sizes $<70 \mu \mathrm{m}$ and a porosity of $83 \%$ (Hapke and Sato 2016). Both layers have been treated, in the model, as continuous matter. Note that we fix the properties of the assumed upper (dust) layer and do not investigate how the results converge to the thermal response of the subsurface if both layer's thermal properties converge.

The bedrock thermal conductivity is a typical $\sim 250 \mathrm{~K}$ value for carbonaceous meteorite analogues (Cold Bokkeveld (CM): $0.5 \mathrm{~W} / \mathrm{m} / \mathrm{K}$, NWA5515 (CK): $1.5 \mathrm{~W} / \mathrm{m} / \mathrm{K}$ (Opeil et al. 2010; Opeil et al. 2012)), while the thermal conductivity of the coarse regolith and dust have been calculated using the model of Sakatani et al. 2017, evaluating conductivity at a mean temperature of $300 \mathrm{~K}$. For the specific heat capacity, values for lunar regolith at $215 \mathrm{~K}$ have been assumed, which is close to the average temperatures we find in our study. Note that our model implicitly assumes that the dust layer is optically and thermally thick (opaque), i.e., the layer thickness $\delta$ must be large compared to the median grain diameter; otherwise, one would need to solve the fully time-dependent system of the radiative and heat transfer equations (Hale and Hapke 2002). The model is therefore only strictly applicable for $\delta>$ at least 5 monolayers of dust particles; thus, very small dust layer depths implicitly require very fine dust for the results to be correct. This can be achieved for C-type dust of grain sizes between 0.1 and $70 \mu \mathrm{m}$ with a porosity varying between 70 and $81 \%$ (calculated with Sakatani et al. 2017).

To put the considered layer thicknesses into perspective, we have furthermore calculated the thermal skin depths associated with Ryugu's orbital and diurnal periods, where the skin depth is defined by

$$
s=\sqrt{k P / \pi \rho c_{p}}
$$

Here, $P$ refers to the orbital and diurnal periods, respectively, and resulting skin depths for the different materials considered are summarized in Table 2.

In the following, we will estimate the influence of a thin dust cover on the surface temperature, and this will be quantified by estimating the apparent thermal inertia of the dust-covered surface, i.e., the thermal inertia that would be obtained from the measured temperature curves under the assumption that the regolith is indeed homogeneous rather than layered. To derive apparent

Table 2 Diurnal ( $\left.s_{\text {diurnal }}\right)$ and annual ( $\left.s_{\text {annual }}\right)$ skin depths for bedrock, coarse regolith, and dust, assuming material parameters as given in Table 1. "Porous rock" and "coarse regolith" are synonyms in this paper

\begin{tabular}{lll}
\hline Material & $s_{\text {diurnal }}[\mathrm{m}]$ & $s_{\text {annual }}(\mathrm{m})$ \\
\hline Bedrock & 0.0790 & 3.05 \\
Porous rock & 0.0270 & 1.04 \\
Dust & $0.0092_{4}$ & 0.357 \\
\hline
\end{tabular}


thermal inertia, four methods are applied, and these will be evaluated in the following. First, thermal inertia can be estimated from the maximum daytime temperature, assuming that insolation is known. Second, the minimum nighttime temperature may be used. Third, the phase shift of the maximum temperature with respect to local noon can be evaluated. And finally, one can fit (in a least-squares sense) part of (e.g., only daytime or nighttime) or even the entire diurnal temperature curve to a homogeneous model. The difference between the thermal inertia of the underlying material and the calculated apparent thermal inertia then indicates how the dust layer changes thermal emission of the base material.

To eliminate sampling effects and rounding errors when estimating thermal inertia from the maximum daytime temperature and phase shift, the temperature curves calculated by solving Eqs. 2 and 3 were output at a resolution of $1^{\circ}$ in rotation angle and then fitted in a window of $\pm 45^{\circ}$ around the maximum by recursive orthogonal polynomials. The optimal degree of the polynomials was determined according to Akaike's information criterion (Akaike 1974) and was found to vary between 6 and 10. Maximum temperature $T_{\max }$ as well as its phase shift $\phi$ with respect to local noon was then read off from the polynomial fit. The daily minimum temperature $T_{\min }$ was determined by taking the global minimum of the $1^{\circ}$ resolution $T(t)$ curves directly.

For the determination of apparent thermal inertia, values obtained from the diurnal temperature curves using the above methods have been compared to those for homogenous surfaces, with $\Gamma$ being varied between 25.3 and $1183 \mathrm{~J} \mathrm{~m}^{-2} \mathrm{~K}^{-1} \mathrm{~s}^{-1 / 2}$. Note that while all quantities derived here depend on insolation and therefore on latitude and season, we have restricted the present analysis to the actual spin and orbital parameters of Ryugu on Aug 3, 2018, and all curves are evaluated at the geographical latitude of MASCOT's landing site. The curves $\phi(\log (\Gamma)), \phi(\log (\Gamma))$, and $\phi(\log (\Gamma))$ are strictly monotonic and have been fit by empirical functions (distorted logistic curves) in $\log (\Gamma)$; root-mean-square residuals are $0.013^{\circ}, 0.019 \mathrm{~K}$, and $0.1 \mathrm{~K}$ for $\phi, T_{\max }$, and $T_{\min }$, respectively, and the functions are physically reasonable when $\Gamma$ tends to zero or infinity. We will need them later to derive the apparent $\Gamma$ from layered model phase lag, minimum and maximum temperatures. We also define, for later uncertainty estimations, a worst-case modeling temperature bias curve as a piecewise linear curve of temperature bias versus rotation angle, given by the nodes $[-180,-94,-86,+93,+$ $179]^{\circ}, \pm[0.35,1.0,0.1,-0.1,0.35] \mathrm{K}$.

One of the common approaches for the interpretation of thermal infrared data is to use a thermal model to determine $\Gamma$ from a best fit to the data. In general, single layer, homogenous models are used to determine the surface thermal inertia, and it is thus essential to understand what thermal inertia one would retrieve if the observed surface was covered by a fine dust layer. Having calculated diurnal temperature curves for dustcovered bedrock and porous rock/regolith, respectively, we have now applied four methods to estimate what we call apparent thermal inertia $\Gamma_{\text {eff }}$, i.e., the thermal inertia one would obtain by inverting or best fitting (in a particular sense) the two-layer model's temperature data using a one-layer model. Apparent thermal inertia has been determined here from phase lag, maximum temperature, minimum temperature, a least-squares fit to the entire diurnal temperature curve, and from a least-squares fit to the daytime and nighttime diurnal temperature curve.

In detail, we proceeded as follows: for the leastsquares fits, an interpolating function for surface temperatures of our homogeneous model for arbitrary $25<\Gamma<1200 \mathrm{~J} \mathrm{~m}^{-2} \mathrm{~K}^{-1} \mathrm{~s}^{-1 / 2}$ was prepared. It interpolates the calculated curves (for 10 discrete values of $\log (\Gamma)$ ) to the desired $\log (\Gamma)$, spline-interpolation). The $X^{2}(\Gamma)$, that is, the sum of the squared deviations between the actual two-layer temperatures and the interpolated homogeneous temperatures, is minimized to find $\Gamma_{\text {eff. }}$ The fit is extended over all rotation angles for the day+night method, for rotation angles from $-93^{\circ}$ to $+93^{\circ}$ for the day only method, and over $\left[-180 . .-94,+93 . .+179^{\circ}\right]$ for the night only method. The accuracy of the calculated $\Gamma_{\text {eff }}$ depends mostly on the interpolation for the homogeneous models; comparing spline to pchip (Piecewise Cubic Hermite Interpolating Polynomial) to a linear interpolation in $\log (\Gamma)$ as the worst case, relative uncertainties in $\Gamma_{\text {eff }}$ are estimated as typically $<1 \%$ with somewhat larger uncertainties for $\delta=3 . .5 \mathrm{~mm}$, where the one- and two-layer curves have their largest discrepancies. To find a conservative uncertainty estimate of $\Gamma_{\text {eff }}$, we add a worst-case modeling temperature bias curve to the nominal 2-layer surface temperature and find the corresponding change in $\Gamma_{\text {eff. }}$.

For the remaining methods, based on the two-layer phase lag, maximum and minimum temperature, we just determine the intersection of the fit functions in $\phi, T_{\max }$, and $T_{\min }$ of our homogeneous calculations with the twolayer values. As the fit functions are strictly monotonous, this gives a unique value each. Based on the rms residuals of the fit functions in $\phi, T_{\max }$, and $T_{\min }$ of our homogeneous calculations plus the uncertainty from our worst-case modeling temperature bias curve (and its derivative at noon), we estimate the precision of the calculated apparent thermal inertia as $0.2 \%$ (lag, $\left.\mathrm{T}_{\max }\right)$ to $5 \%\left(\mathrm{~T}_{\min }\right)$.

\section{Results and discussion}

To demonstrate the influence of dust on thermal emission, we have calculated example diurnal temperature 
curves for the cases of dust-covered bedrock and regolith by solving Eqs. 2 and 3, for a dust layer thickness of $\delta=1 \mathrm{~mm}$. They are shown in Fig. 2. As is evident from the figure, at this dust thickness, the dust layer masks the thermal emission of the underlying material already almost completely, and both diurnal curves are hardly distinguishable. Only small differences in nighttime temperature and cooling rate after sunset remain, but these are generally below $10 \mathrm{~K}$. Overall, curves resemble those of low thermal inertia units, i.e., they exhibit small phase lag $\phi$, high $T_{\max }$, and have a symmetric appearance. The influence of the bottom layer is most visible shortly after sunset, when the fast cooling rate suddenly decreases, giving rise to a "kink" in the temperature curveand resulting in night-time temperatures which are significantly higher than for the homogeneous case. The cooling rate at night is significantly lower (and almost vanishes for intermediate dust thicknesses) than in the homogeneous case.

To study the influence of layer thickness on the shape of the diurnal temperature curve, we have calculated surface temperatures varying the dust layer thicknesses $\delta$ between 0 and $100 \mathrm{~mm}$. Results of these calculations are summarized in Fig. 3, where phase lag $\phi$ of the maximum daytime temperature with respect to local noon, the maximum temperature $T_{\max }$, as well as the minimum temperature $T_{\mathrm{min}}$, are shown as a function of $\delta$. Here, the red line corresponds to the model of dust covered regolith/porous rock, while the blue line corresponds to the bedrock case. In addition, dashed horizontal lines indicate $\phi, T_{\max }$, and $T_{\min }$ which correspond to homogeneous dust, regolith, and bedrock, respectively.

In Fig. 3, $\phi, T_{\max }$, and $T_{\min }$ vary smoothly as a function of $\delta$, and limiting values correspond to those for the homogeneous case for small layer thicknesses, while curves converge to the values corresponding to a homogeneous dust layer for large thicknesses as expected. For thicknesses greater than about a diurnal skin depth, the upper layer becomes thermally "opaque" and the model results are indistinguishable from the constant property case (for a full orbit, $\delta$ needs to be greater than a seasonal skin depth, $\sim 36 \mathrm{~mm}$, to converge to the constant property case).

For the phase lag, the strongest change with dust cover occurs at a layer thickness close to $\delta=0.1 \mathrm{~mm}$ for dustcovered bedrock and $\delta=0.3 \mathrm{~mm}$ for dust-covered regolith, respectively. A peculiar feature is the region between about $1 \mathrm{~mm} \leq \delta \leq 15 \mathrm{~mm}$, where the thermal lag is significantly smaller than the value for homogeneous dust. This effect is discussed below. For $T_{\max }$ and $T_{\min }$, the strongest influence is seen around $\delta=1 \mathrm{~mm}$. Noticeable effects already occur for extremely small amounts of dust, and the results converge to pure dust properties for large thicknesses: using the $10 \%$ and $90 \%$ amplitudes, the change of lag angle occurs between layer thicknesses 0.01 and $0.4 \mathrm{~mm}$ for dust on bedrock and 0.04 and $0.9 \mathrm{~mm}$ for dust on coarse regolith. The change of $T_{\max }$ occurs between layer thicknesses 0.07 and $3 \mathrm{~mm}$ for dust on bedrock and 0.2 and $5 \mathrm{~mm}$ for dust on coarse regolith and the change of $T_{\min }$ occurs between layer thicknesses 0.2
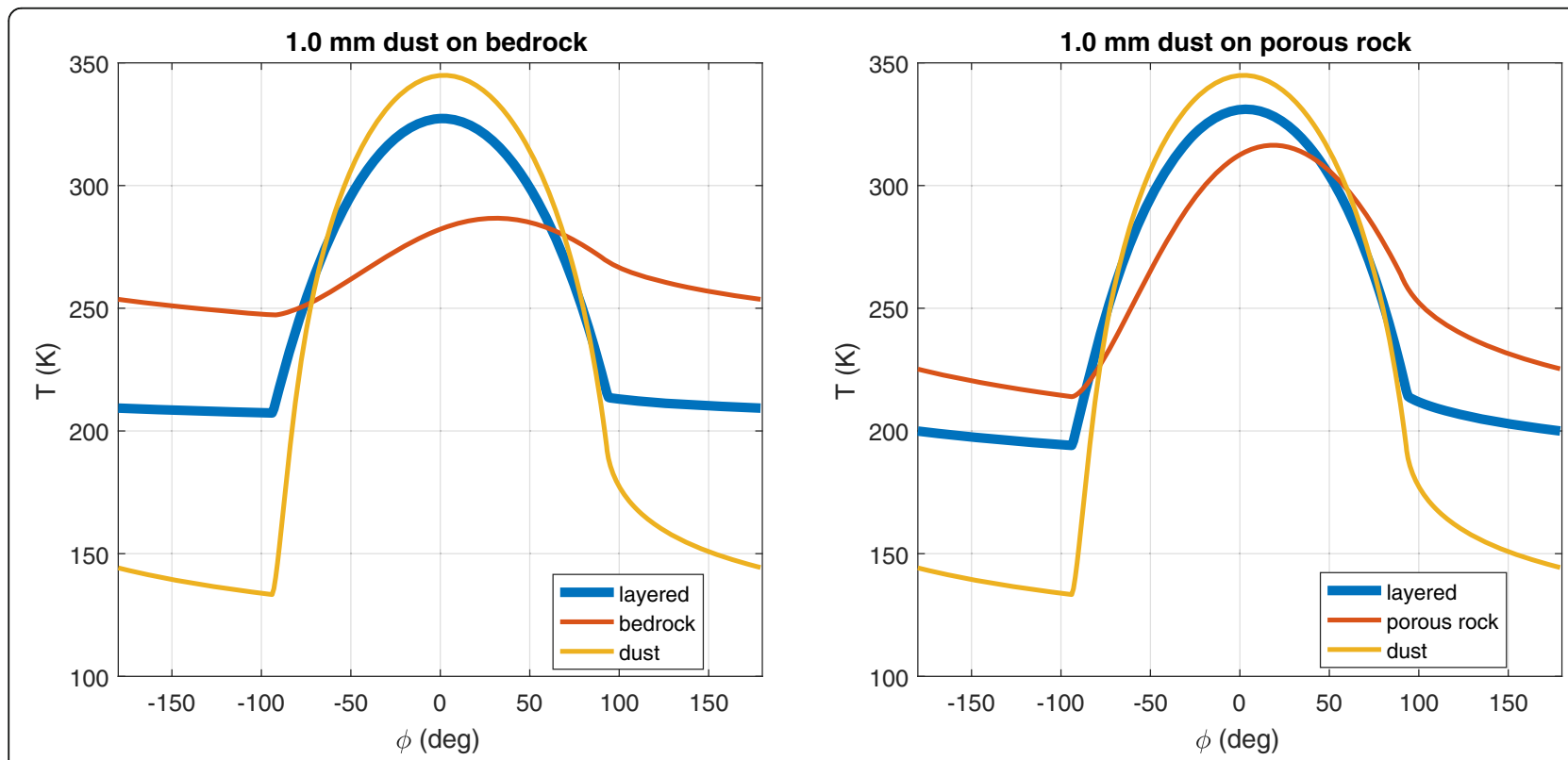

Fig. 2 Left: Example diurnal temperature curve (bold line) for bedrock covered with $1 \mathrm{~mm}$ of dust. Right: same as left, but for porous rock/coarse regolith covered with $1 \mathrm{~mm}$ of dust. The surface temperature curves for the homogeneous dust and subsurface material are shown for comparison 

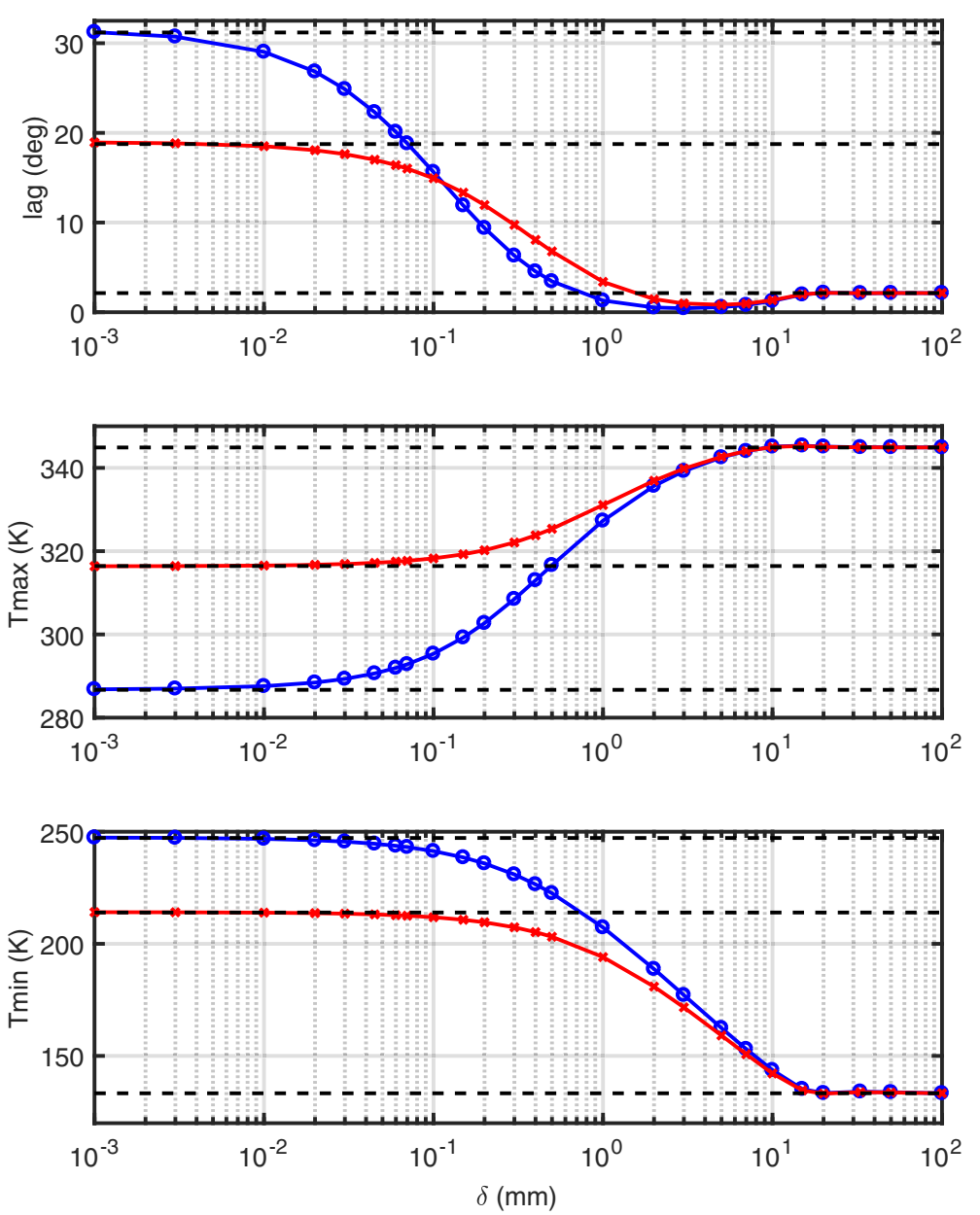

Fig. 3 Phase lag $\varphi$, maximum diurnal temperature $T_{\max }$ and minimum diurnal temperature $T_{\min }$ as a function of dust thickness $\delta$. The red fit curve corresponds to the case of dust on coarse regolith, whereas the blue fit curve corresponds to dust on bedrock. Broken horizontal lines represent $\varphi, T_{\max }$ and $T_{\min }$ for the homogeneous cases of dust, regolith, and bedrock, respectively

and $9 \mathrm{~mm}$ for dust on bedrock and 0.4 and $10 \mathrm{~mm}$ for dust on coarse regolith. We see that phase lag is most sensitive to a surface dust layer and minimum temperature is least sensitive.

Results for apparent thermal inertia are summarized in Fig. 4. Already an extremely thin dust layer is found to significantly alter the retrieved apparent thermal inertia, which furthermore depends significantly on the applied inversion method. Out of the tested methods, determination of thermal inertia from lag is particularly sensitive to dust, whereas the estimation based on minimum temperature is least affected. However, even in this best case, apparent thermal inertia starts to significantly deviate ( $>7 \%$ for the porous rock case, $25 \%$ for the bedrock case) from the background value for dust layer thicknesses above $\delta>0.1 \mathrm{~mm}$, corresponding to only 100th of a diurnal skin depth. Apparent thermal inertia converges to that of the surface dust once layer thicknesses corresponding to 1-2 diurnal skin depths $(9-18 \mathrm{~mm})$ are reached.
As may be expected, the masking effect is more pronounced for the high thermal inertia bedrock, and a $1 \mathrm{~mm}$ dust layer will completely mask the underlying bedrock if $\Gamma_{\text {eff }}$ is based on the phase lag of the maximum diurnal temperatures. Furthermore, even if the estimate is based on minimum diurnal temperatures, apparent thermal inertia will still be changed from 1183 to $241 \mathrm{~J} \mathrm{~m}^{-2} \mathrm{~K}^{-1} \mathrm{~s}^{-1 / 2}$. Similarly, the apparent thermal inertia of porous rock will be changed from $300 \mathrm{~J} \mathrm{~m}^{-2} \mathrm{~K}^{-1} \mathrm{~s}^{-1 / 2}$ to $41 \mathrm{~J} \mathrm{~m}^{-2} \mathrm{~K}^{-1} \mathrm{~s}^{-1 / 2}$ and $163 \mathrm{~J} \mathrm{~m}^{-2} \mathrm{~K}^{-1} \mathrm{~s}^{-1 / 2}$ for $1 \mathrm{~mm}$ dust using these methods, respectively. All other methods yield intermediate results with the method based on phase lag being the most sensitive to dust coverage.

Example diurnal temperature curves and six model curves for the case $\delta=0.4 \mathrm{~mm}$ are shown in Fig. 5 for the bedrock and regolith case, respectively. This corresponds to the minimum physically realistic layer thickness for the chosen dust grain size. Temperature curves as calculated from the two-layer model (true) are shown in blue, and 


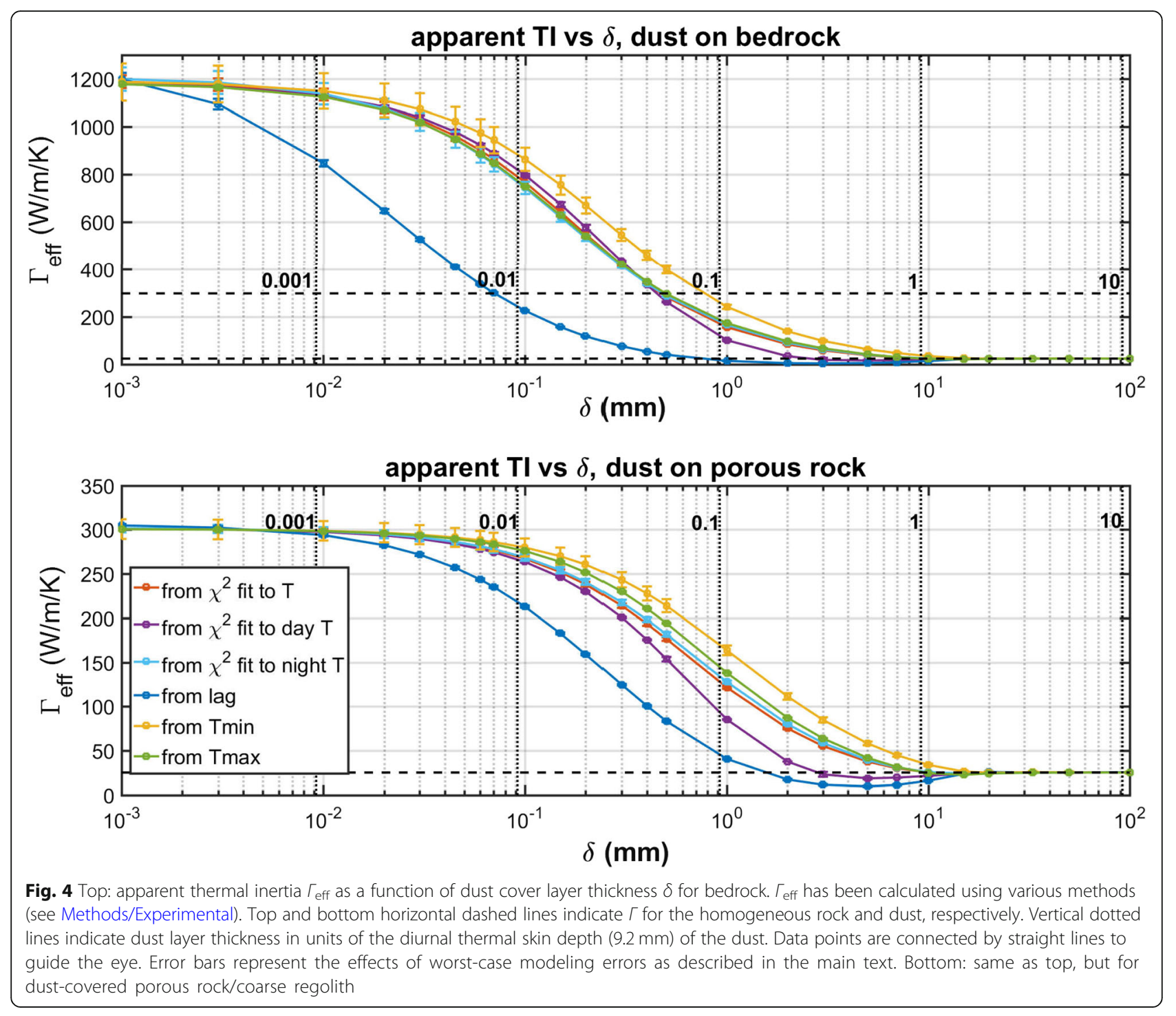

best fitting model curves as derived assuming a homogeneous one-layer model are shown for comparison. As is evident from the figure, all one-layer model curves exhibit large misfits with respect to the 2-layer model, even if they are capable of fitting phase lag, maximum, and minimum temperatures, respectively. Systematic deviations of several tens of $\mathrm{K}$ are common, in particular during daytime and shortly after sunset. The least successful model in reproducing the diurnal temperature curve is the one based on the inversion of the thermal lag, which is most severely affected by the presence of dust. Also note that none of the models reproduces the peculiar kink of the two-layer curve immediately after sunset, which is characteristic for our low-over-high thermal inertia vertical layering.

The result of a phase lag that is even smaller than the one for pure dust (for dust thicknesses of $\sim 0.1$ to $\sim 1$ dust diurnal skin depth) is surprising and it deserves an explanation. We tried hard to explain the anomaly with numerical errors: after all, in the relevant $\delta$ range, the difference between noon and maximum temperature is much smaller than $0.1 \mathrm{~K}$, the estimated absolute accuracy of our thermal model. The determination of the maximum, hence the thermal lag (phase of the diurnal maximum relative to local noon), then solely depends on fitting the model temperature curve with the optimum degree recursive orthogonal polynomial as described above, $\pm 45^{\circ}$ around noon. However, numerical experiments showed that a $0.1 \mathrm{~K}$ Gaussian noise on the model temperatures can only change the retrieved phase lag by $<0.1^{\circ}$; the only relevant influence is by a time-varying temperature bias. Analytically, the bias in phase lag produced by a slope $\mathrm{d} T_{\text {bias }} / \mathrm{d} x$ in the model temperature is $\delta \phi=\left(\mathrm{d} T_{\text {bias }} / \mathrm{d} x\right) / \mathrm{d}^{2} T / \mathrm{d} x^{2}$ ( $x$ : rotation angle in ${ }^{\circ}, \mathrm{d}^{2} T / \mathrm{d} x^{2}$ curvature of the surface temperature at the maximum, typically $-0.025 \mathrm{~K} /{ }^{2}$ 

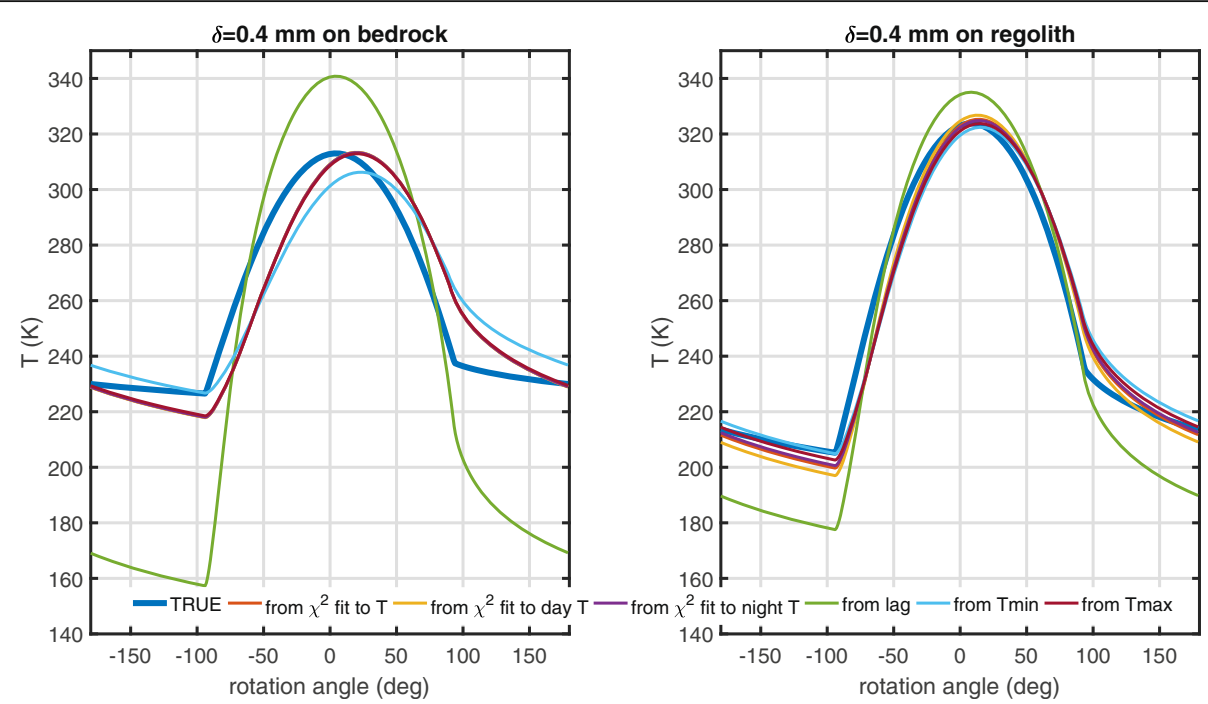

Fig. 5 Left: temperature as a function of rotation angle for the case of bedrock covered by a $0.4 \mathrm{~mm}$ dust layer (true, blue curve) along with 6 best-fitting models calculated under the assumption of a homogeneous one layer model. Right: same as top, but for dust-covered regolith

for the anomalous lag range). To explain the anomaly, e.g., for $\delta=3 \mathrm{~mm}$ on bedrock, we thus would need a $\mathrm{d} T_{\text {bias }} / \mathrm{d} x$ of $>0.05 \mathrm{~K} /{ }^{\circ}$, that is, $2.2 \mathrm{~K}$ over the course of $45^{\circ}$ in rotation angle ( $1 \mathrm{~h}$ in time). This is not consistent with our analysis of the numerical accuracy of the thermal model: calculating the case of $3 \mathrm{~mm}$ dust on bedrock both in standard resolution and with much increased resolution in the time and depth grids, we find a bias between -0.026 and $+0.06 \mathrm{~K}$, and a slope at noon $\pm 45^{\circ}$ of just $4 \times 10^{-5} \mathrm{~K} /{ }^{\circ}$. This can bias the phase lag by only $0.002^{\circ}$. Our worst-case modeling temperature bias curve (see above), which has been used to calculate the error bars in Fig. 4, has a $\mathrm{d} T_{\text {bias }} / \mathrm{d} x$ of $-0.0011 \mathrm{~K} /{ }^{\circ}$ and can change the phase lag by $0.05^{\circ}$ at most.

So, we have to conclude that this phase lag anomaly might be a real, physical effect.

Qualitatively, for $\delta$ in the range of 0.1 to 1 diurnal skin depths, the subsurface will store heat at night (compare the very small cooling rate at night in Fig. 5). After sunrise, the subsurface is still warm and the heat flux from stored heat adds up to the daytime heat flux from insolation, the combined effect of which is a maximum temperature significantly earlier than expected for a homogeneous dust surface with no upwelling heat.

How likely is a dust layer on the surface of a small body? Due to the low gravity, which is close to only $1.5 \times 10^{-4} \mathrm{~m} \mathrm{~s}^{-2}$ on Ryugu and corresponds to an escape velocity of only $0.4 \mathrm{~m} \mathrm{~s}^{-1}$, small asteroids can hardly keep fines produced during impacts. However, not all fine particles necessarily reach escape velocity, and lofted particles may re-accumulate on the surface as so-called air-fall. On the other hand, seismic accelerations during impact events may reach $0.1 \mathrm{~m} \mathrm{~s}^{-2}$ at distances of $200 \mathrm{~m}$ from the impact site (Garcia et al. 2015) and can thus be a factor of one thousand larger than gravity. As the granular bond number (Scheeres et al. 2010), which is defined as the ratio between van der Waals forces and the weight of the particle, is larger than 1 for dust particles smaller than $0.1 \mathrm{~m}$ under Ryugu gravity conditions, only dust particles $<0.1 \mathrm{~mm}$ are expected to stick during large impacts. Such particles could for example originate from micrometeorite impacts, solar wind sputtering, or thermal fatigue (Delbo et al. 2014; Szalay et al. 2018) and could cover coarser pebbles or bedrock surfaces. It therefore seems possible that such fines could be present on small airless bodies.

\section{Conclusions}

We have calculated the influence of dust layers on the surface temperature on airless bodies by solving the heat conduction equation for a simple two-layer model. Results indicate that already a very thin layer of dust on top of ordinary millimeter- to centimeter-sized regolith, porous rock, or compact bedrock has a significant systematic influence on the observed surface temperatures. When interpreting these temperatures in terms of homogeneous thermophysical properties, the derived apparent thermal inertia is neither representative for the surface nor the subsurface layer for a wide range of dust layer thicknesses. Masking of subsurface thermal properties starts already at dust thicknesses as low as 0.01 diurnal skin depths, while the dust becomes thermally opaque for layer thicknesses in excess of two skin depths. This is qualitatively a similar result as reported by Paige et al. 1994 and Putzig and Mellon 2007, but 
our results suggest that significant and nearly complete masking occurs already at much smaller layer thicknesses for our layer model.

This effect occurs for all the methods applied to estimate thermal inertia using homogeneous models. While using the thermal phase lag of the maximum daytime temperature to arrive at apparent $\Gamma$ results in the most erroneous thermal inertia estimates, all methods suffer from the principle problem that even small dust layers significantly alter the thermal characteristics of the surface, which cannot be adequately modeled using a single homogeneous layer.

A dust-covered surface should be readily detectable in the diurnal temperature curve, which would show the characteristic kink of the temperature curve shortly after sunset for intermediate $\delta$. Furthermore, the large fitting residuals when trying to invert the data using a one-layer model should also indicate that two layer models are required to properly fit the data. Then, data should be inverted using a thermal model with depth-dependent thermophysical properties without relying on thermal inertia if indications for a dust-covered surface exist. Such a model will require at least two layers and 5 free parameters, i.e., $k_{\text {dust }}$, $k_{\text {reg }}, \rho c_{\mathrm{p} \text {,dust }}, \rho c_{\mathrm{p} \text {,reg, }}$, as well as the dust layer thickness $\delta$. If a priori knowledge is available as, e.g., for the Moon, some of these parameters may be fixed to reduce the complexity of the inversion (Vasavada et al. 1999), but this is generally lacking for asteroids. Therefore, all five parameters are required since the concept of thermal inertia breaks down if thermophysical properties are not invariant with depth. However, the overall accuracy and uniqueness of such an inversion with noise has yet to be determined.

High-resolution images returned from the surface of Ryugu show no indications for any appreciable dust layer on the surface of the rock observed by MASCOT (Jaumann, R. et al.: In-situ investigation of asteroid (162173) Ryugu by the Mobile Asteroid Surface Scout (MASCOT) Camera (MASCam), submitted to Science, 2019). The diurnal temperature curve as observed by the MASCOT radiometer MARA can be well explained by a homogeneous $\Gamma \approx 280 \mathrm{~J} \mathrm{~m}^{-2} \mathrm{~K}^{-1} \mathrm{~s}^{-1 / 2}$. As there is also no kink discernible at sunset (Grott et al. 2019), this indicates that dust plays an insignificant role in the thermal emission from the asteroid.

\section{Acknowledgements}

Organization of stimulating international scientific exchange in the Hayabusa2 IRSG (International Regolith Science Group) by JAXA is gratefully acknowledged.

\section{Authors' contributions}

JB proposed the topic, conceived and designed the study, and analyzed the simulated data sets. EK carried out the thermal model numerical calculations. MG and MH added to the scientific discussion of results. HS and TA checked the calculations with their own codes and provided important comments. NS,
$\mathrm{KO}$, and TO discussed models and provided background on the TIR instrument. All authors read and approved the final manuscript.

\section{Funding}

KO was supported by the JSPS Core-to-Core Program "International Network of Planetary Sciences". NS and TO are supported by the JSPS Kakenhi No. JP17H06459 (Aqua Planetology).

\section{Availability of data and materials}

Simulation data is available from the corresponding author upon reasonable request.

\section{Competing interests}

The authors declare that they have no competing interest.

\section{Author details}

${ }^{1}$ DLR - German Aerospace Center, RB-MUSC, 51147 Cologne, Germany. ${ }^{2}$ DLR - German Aerospace Center, Institute for Planetary Research, 12489 Berlin, Germany. ${ }^{3}$ Planetary Exploration Research Center, Chiba Institute of Technology, Chiba 275-0016, Japan. Institute of Space and Astronautical Science, Japan Aerospace Exploration Agency, 3-1-1 Yoshinodai, Chuo-ku, Sagamihara, Kanagawa 252-5210, Japan. ${ }^{5}$ Department of Planetology, Kobe University, 1-1 Rokkodaicho, Nada, Kobe, Hyogo 657-8501, Japan. ${ }^{6}$ Division of System and Information Engineering, Ashikaga University, 286-1 Omae-cho, Ashikaga-shi, Tochigi 326-8558, Japan.

Received: 24 September 2018 Accepted: 27 May 2019

Published online: 08 July 2019

\section{References}

Akaike H (1974) A new look at the statistical model identification. IEEE Trans Autom Control 19(6):716-723

Audouard J, Poulet F, Vincendon M, Bibring JP, Forget F, Langevin Y, Gondet B (2014) Mars surface thermal inertia and heterogeneities from OMEGA/MEX. Icarus 233:194-213

Biele J, Grott M (2018) Reference heat capacity for asteroid regolith from 10 to 1000K. In: 49th Lunar and Planetary Science Conference. Lunar and Planetary Institute: Abstract \#1877, Houston

Consolmagno G, Britt D, Macke R (2008) The significance of meteorite density and porosity. Chemie der Erde-Geochemistry 68(1):1-29

Delbo M, Libourel G, Wilkerson J, Murdoch N, Michel P, Ramesh KT, Ganino C, Verati C, Marchi S (2014) Thermal fatigue as the origin of regolith on small asteroids. Nature 508(7495):233-236

Delbo M, Mueller M, Emery JP, Rozitis B, Capria MT (2015) In: Michel P, DeMeo F, Bottke WF (eds) Asteroid thermophysical modeling, Asteroids IV. The University of Arizona Press, Tucson, pp 107-128

Ditteon R (1982) Daily temperature variations on Mars. J Geophys Res Solid Earth 87(B12):10197-10214

Edwards CS, Piqueux S, Hamilton VE, Fergason RL, Herkenhoff KE, Vasavada AR, Bennett KA, Sacks L, Lewis K, Smith MD (2018) The thermophysical properties of the Bagnold dunes, Mars: ground-truthing orbital data. J Geophys Res Planets 123(5):1307-1326

Garcia RF, Murdoch N, Mimoun D (2015) Micro-meteoroid seismic uplift and regolith concentration on kilometric scale asteroids. Icarus 253:159-168

Grott M, Knollenberg J, Hamm M, Ogawa K, Jaumann R, Otto K, Delbo M, Michel P, Biele J, Neumann W, Knapmeyer M, Kührt E, Senshu H, Okada T, Helbert J, Maturilli A, Müller N, Hagermann A, Sakatani N, Tanaka S, Arai T, Mottola S, Tachibana S, Pelivan I, Drube L, Vincent J-B, Yano H, Pilorget C, Matz KD, Schmitz N, Koncz A, Trauthan F, Schlotterer M, Krause C, Ho T-M, Moussi-Soffys A (2019) Low thermal conductivity boulder with high porosity identified on C-type asteroid (162173) Ryugu using MARA - the MASCOT radiometer. Nature Astronomy submitted. https://doi.org/10. 1038/s41550-019-0832-x

Hale A, Hapke B (2002) A time-dependent model of radiative and conductive thermal energy transport in planetary regoliths with applications to the moon and mercury. Icarus 156(2):318-334

Hapke B, Sato H (2016) The porosity of the upper lunar regolith. Icarus 273:75-83

Harris AW, Drube L (2016) Thermal tomography of asteroid surface structure. Astrophys J 832(2):127

Hayne PO, Bandfield JL, Siegler MA, Vasavada AR, Ghent RR, Williams JP, Greenhagen BT, Aharonson O, Elder CM, Lucey PG (2017) Global regolith 
thermophysical properties of the moon from the Diviner Lunar Radiometer Experiment. J Geophy Res Planets 122:2371-2400. https://doi.org/10.1002/ 2017JE005387.

Ho T-M, Baturkin V, Grimm C, Grundmann JT, Hobbie C, Ksenik E, Lange C, Sasaki K, Schlotterer M, Talapina M, Temtanasombat N, Wejmo E, Witte L, Wrasmann M, Wübbles G, Rößler J, Ziach C, Findlay R, Biele J, Krause C, Ulamec S, Lange M, Mierheim O, Lichtenheldt R, Maier M, Reill J, Sedlmayr H-J, Bousquet P, Bellion A, Bompis O, Cenac-Morthe C, Deleuze M, Fredon S, Jurado E, Canalias E, Jaumann R, Bibring J-P, Glassmeier KH, Herčík D, Grott M, Celotti L, Cordero F, Hendrikse J, Okada T (2017) MASCOT_the mobile asteroid surface scout onboard the Hayabusa2 mission. Space Sci Rev 208:339-374

Jakosky BM (1979) The effects of nonideal surfaces on the derived thermal properties of Mars. J Geophys Res Solid Earth 84(B14):8252-8262

Jaumann R, Schmitz N, Koncz A, Michaelis H, Schroeder SE, Mottola S, Trauthan F, Hoffmann H, Roatsch T, Jobs D, Kachlicki J, Pforte B, Terzer R, Tschentscher M, Weisse S, Mueller U, Perez-Prieto L, Broll B, Kruselburger A, Ho T-M, Biele J, Ulamec S, Krause C, Grott M, Bibring J-P, Watanabe S, Sugita S, Okada T, Yoshikawa M, Yabuta H (2017) The camera of the MASCOT asteroid lander on board Hayabusa 2. Space Sci Rev 208(1-4):375-400

Keihm S, Langseth M Jr (1973) Surface brightness temperatures at the Apollo 17 heat flow site: thermal conductivity of the upper $15 \mathrm{~cm}$ of regolith. In: Lunar and Planetary Science Conference Proceedings

Kührt E, Giese B (1989) A thermal model of the Martian satellites. Icarus 81(1):102-112

Mellon M, Putzig N (2007) The apparent thermal inertia of layered surfaces on Mars. In: Lunar and planetary science conference

Mellon MT, Feldman WC, Prettyman TH (2004) The presence and stability of ground ice in the southern hemisphere of Mars. Icarus 169(2):324-340

Mitchell DL, De Pater I (1994) Microwave imaging of Mercury's thermal emission at wavelengths from 0.3 to $20.5 \mathrm{~cm}$. Icarus 110(1):2-32

Opeil CP, Consolmagno GJ, Britt DT (2010) The thermal conductivity of meteorites: new measurements and analysis. Icarus 208(1):449-454

Opeil CP, Consolmagno GJ, Safarik DJ, Britt DT (2012) Stony meteorite thermal properties and their relationship to meteorite chemical and physical states. Meteorit Planet Sci 47:319-329

Paige DA, Bachman JE, Keegan KD (1994) Thermal and albedo mapping of the polar regions of Mars using Viking thermal mapper observations: 1. North polar region. J Geophys Res Planets 99(E12):25959-25991

Pelivan I (2018) Thermophysical modelling for high-resolution digital terrain models. Mon Not R Astron Soc 478(1):386-398

Putzig NE, Mellon MT (2007) Apparent thermal inertia and the surface heterogeneity of Mars. Icarus 191(1):68-94

Ruff SW, Christensen PR (2002) Bright and dark regions on Mars: particle size and mineralogical characteristics based on thermal emission spectrometer data. J Geophys Res Planets 107(E12)Vol. 107, E12, 5127, pages 10-1 to 10-22

Sakatani N, Ogawa K (2017) Temperature calculation of coarse-grained regolith surface on small bodies. JpGU-AGU Joint Meeting, May 20 - May 25, 2017. JpGU, Tokyo

Sakatani N, Ogawa K, lijima Y, Arakawa M, Honda R, Tanaka S (2017) Thermal conductivity model for powdered materials under vacuum based on experimental studies. AIP Adv 7(1):015310

Scheeres DJ, Hartzell CM, Sánchez P, Swift M (2010) Scaling forces to asteroid surfaces: the role of cohesion. Icarus 210(2):968-984

Sugita S, Honda R, Morota T, Kameda S, Sawada H, Tatsumi E, Yamada M, Honda C, Yokota Y, Kouyama T, Sakatani N, Ogawa K, Suzuki H, Okada T, Namiki N, Tanaka S, lijima Y, Yoshioka K, Hayakawa M, Cho Y, Matsuoka M, Hirata N, Hirata N, Miyamoto H, Domingue D, Hirabayashi M, Nakamura T, Hiroi T, Michikami T, Michel P, Ballouz R-L, Barnouin OS, Ernst CM, Schröder SE, Kikuchi H, Hemmi R, Komatsu G, Fukuhara T, Taguchi M, Arai T, Senshu H, Demura H, Ogawa Y, Shimaki Y, Sekiguchi T, Müller TG, Hagermann A, Mizuno T, Noda H, Matsumoto K, Yamada R, Ishihara Y, Ikeda H, Araki H, Yamamoto K, Abe S, Yoshida F, Higuchi A, Sasaki S, Oshigami S, Tsuruta S, Asari K, Tazawa S, Shizugami M, Kimura J, Otsubo T, Yabuta H, Hasegawa S, Ishiguro M, Tachibana S, Palmer E, Gaskell R, Le Corre L, Jaumann R, Otto K, Schmitz N, Abell PA, Barucci MA, Zolensky ME, Vilas F, Thuillet F, Sugimoto C, Takaki N, Suzuki Y, Kamiyoshihara H, Okada M, Nagata K, Fujimoto M, Yoshikawa M, Yamamoto Y, Shirai K, Noguchi R, Ogawa N, Terui F, Kikuchi S, Yamaguchi T, Oki Y, Takao Y, Takeuchi H, Ono G, Mimasu Y, Yoshikawa K, Takahashi T, Takei Y, Fujii A, Hirose C, Nakazawa S, Hosoda S, Mori O, Shimada T, Soldini S, Iwata T, Abe M, Yano H, Tsukizaki R, Ozaki M, Nishiyama K, Saiki T, Watanabe S, Tsuda Y (2019) The geomorphology, color, and thermal properties of Ryugu: implications for parent-body processes. Science 364(6437):eaaw0422
Szalay J, Poppe A, Agarwal J, Britt D, Belskaya I, Horányi M, Nakamura T, Sachse M, Spahn F (2018) Dust phenomena relating to airless bodies. Space Sci Rev 214(5):98

Titus T, Prettyman T, Colaprete A (2006) Thermal characterization of the three proposed Phoenix landing sites. In: 37th Annual Lunar and Planetary Science Conference

Vasavada AR, Bandfield JL, Greenhagen BT, Hayne PO, Siegler MA, Williams J-P, Paige DA (2012) Lunar equatorial surface temperatures and regolith properties from the Diviner Lunar Radiometer Experiment. J Geophys Res Planets 117(E12):EOOH18

Vasavada AR, Paige DA, Wood SE (1999) Near-surface temperatures on Mercury and the Moon and the stability of polar ice deposits. Icarus 141(2):179-193

Vasavada AR, Piqueux S, Lewis KW, Lemmon MT, Smith MD (2017) Thermophysical properties along Curiosity's traverse in Gale crater, Mars, derived from the REMS ground temperature sensor. Icarus 284:372-386

Wada K, Grott M, Michel P, Walsh KJ, Barucci AM, Biele J, Blum J, Ernst CM, Grundmann JT, Gundlach B, Hagermann A, Hamm M, Jutzi M, Kim M-J, Kührt E, Le Corre L, Libourel G, Lichtenheldt R, Maturilli A, Messenger SR, Michikami T, Miyamoto H, Mottola S, Müller T, Nakamura AM, Nittler LR, Ogawa K, Okada T, Palomba E, Sakatani N, Schröder SE, Senshu H, Takir D, Zolensky ME, project IRSG i H (2018) Asteroid Ryugu before the Hayabusa2 encounter. Prog Earth Planet Sci 5(1):82

Watanabe S, Hirabayashi M, Hirata N, Hirata N, Noguchi R, Shimaki Y, Ikeda H, Tatsumi E, Yoshikawa M, Kikuchi S, Yabuta H, Nakamura T, Tachibana S, Ishihara Y, Morota T, Kitazato K, Sakatani N, Matsumoto K, Wada K, Senshu H, Honda C, Michikami T, Takeuchi H, Kouyama T, Honda R, Kameda S, Fuse T, Miyamoto H, Komatsu G, Sugita S, Okada T, Namiki N, Arakawa M, Ishiguro M, Abe M, Gaskell R, Palmer E, Barnouin OS, Michel P, French AS, McMahon JW, Scheeres DJ, Abell PA, Yamamoto Y, Tanaka S, Shirai K, Matsuoka M, Yamada M, Yokota Y, Suzuki H, Yoshioka K, Cho Y, Tanaka S, Nishikawa N, Sugiyama T, Kikuchi H, Hemmi R, Yamaguchi T, Ogawa N, Ono G, Mimasu Y, Yoshikawa K, Takahashi T, Takei Y, Fujii A, Hirose C, Iwata T, Hayakawa M, Hosoda S, Mori O, Sawada H, Shimada T, Soldini S, Yano H, Tsukizaki R, Ozaki M, lijima Y, Ogawa K, Fujimoto M, Ho T-M, Moussi A, Jaumann R, Bibring J-P, Krause C, Terui F, Saiki T, Nakazawa S, Tsuda Y (2019) Hayabusa2 arrives at the carbonaceous asteroid 162173 Ryugu-a spinning top-shaped rubble pile. Science 364(6437):268-272

Watanabe S-i, Tsuda Y, Yoshikawa M, Tanaka S, Saiki T, Nakazawa S (2017) Hayabusa2 Mission overview. Space Sci Rev 208(1-4):3-16

\section{Publisher's Note}

Springer Nature remains neutral with regard to jurisdictional claims in published maps and institutional affiliations.

\section{Submit your manuscript to a SpringerOpen ${ }^{\circ}$ journal and benefit from:}

- Convenient online submission

- Rigorous peer review

- Open access: articles freely available online

High visibility within the field

- Retaining the copyright to your article

Submit your next manuscript at $\boldsymbol{\nabla}$ springeropen.com 